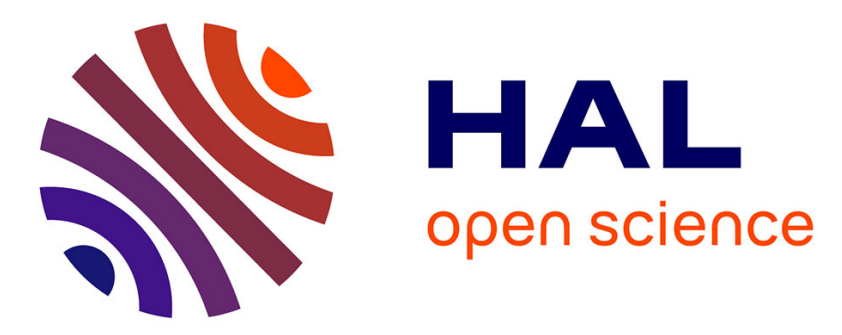

\title{
Optimal input design for parameter estimation of nonlinear systems: case study of an unstable delta wing
} Jun Qian, Madiha Nadri, Pascal Dufour

\section{To cite this version:}

Jun Qian, Madiha Nadri, Pascal Dufour. Optimal input design for parameter estimation of nonlinear systems: case study of an unstable delta wing. International Journal of Control, 2017, 90 (4), pp.873887. 10.1080/00207179.2016.1225990 . hal-01518400

\author{
HAL Id: hal-01518400 \\ https://hal.science/hal-01518400
}

Submitted on 4 May 2017

HAL is a multi-disciplinary open access archive for the deposit and dissemination of scientific research documents, whether they are published or not. The documents may come from teaching and research institutions in France or abroad, or from public or private research centers.
L'archive ouverte pluridisciplinaire HAL, est destinée au dépôt et à la diffusion de documents scientifiques de niveau recherche, publiés ou non, émanant des établissements d'enseignement et de recherche français ou étrangers, des laboratoires publics ou privés. 


\title{
SUBMISSION, SPECIAL ISSUE: IDENTIFICATION AND CONTROL OF NONLINEAR ELECTRO-MECHANICAL SYSTEMS
}

\author{
Optimal input design for parameter estimation of nonlinear systems: \\ case study of an unstable delta wing
}

\author{
J. Qian ${ }^{\mathrm{a}, \mathrm{b}}$, M. Nadri ${ }^{\mathrm{a}}{ }$ and P. Dufour ${ }^{\mathrm{a}}$ \\ ${ }^{a}$ Université de Lyon, Lyon F-69003, Université Lyon 1, CNRS UMR 5007, Laboratory of Process Control \\ and Chemical Engineering (LAGEP), Villeurbanne 69100, France; \\ ${ }^{b}$ Acsystème, 4 rue René Dumont, 35700 Rennes, France \\ (Received 00 Month 20XX; accepted 00 Month 20XX)
}

\begin{abstract}
A closed loop optimal experiment design for on-line parameter identification approach is developed for nonlinear dynamic systems. The goal of the observer and the nonlinear model predictive control theories is here to perform online computation of the optimal time-varying input and to estimate the unknown model parameters online. The main contribution consists in combining Lyapunov stability theory with an existing closed loop identification approach, in order to maximize the information content in the experiment and meanwhile to asymptotically stabilize the closed loop system. To illustrate the proposed approach, the case of an open loop unstable aerodynamic mechanical system is discussed. The simulation results show that the proposed algorithm allows to estimate all unknown parameters, which was not possible according to previous work, while keeping the closed loop system stable.
\end{abstract}

Keywords: closed loop identification; optimal experiment design; model predictive control; stability; nonlinear observer; persistent excitation, delta wing; mechanical system.

\section{Introduction}

Accurate modeling is required for simulation, optimization or control of dynamic processes. Therefore, the identification of unknown model parameters can not be avoided. Badly designed experiments for model parameter identification can be costly (e.g. materials fed at the process inlet, energy consumption during such experiments, output materials with undesired properties) and increase the time needed for pure model identification. Optimal experiment design (OED) is a classical technique for parameter identification purposes (Goodwin et al., 1977; Ljung, 1999). However, in a large part of the existing literature on OED for parameter identification, optimal input design is separated from parameter estimation. In that case, the experimental data is gathered from previous experiences for further offline model parameter estimation (Barz et al., 2012; Ljung, 1999; Walter and Pronzato, 1994). Moreover, for many decades OED for parameter estimation has only been applied to linear or approximated linearized models (Franceschini and Macchietto, 2008; Keviczky, 1975; Ng et al., 1977), whereas in areas such as biological and chemical processes, models are highly nonlinear, even sometimes unstable.

Recently, coupled closed loop OED and online parameter estimation approaches have been developed by several authors for nonlinear multivariable stable systems. The basic objective in this kind of approach is to maximize the information content of the experiments to improve the ac-

${ }^{*}$ Corresponding author. Email: nadri@lagep.univ-lyon1.fr 
curacy of parameter estimation, which is usually described by a sensitivity criterion based on the Fisher information matrix (FIM)(Goodwin et al., 1977; Ljung, 1999; Walter and Pronzato, 1994). In (Jayasankar et al. , 2010), the authors developed an OED for on-line parameter estimation in the multivariable case but without any concern with the closed loop stability. In (Zhu and Huang, 2011), the authors used steady state analysis to add linear equality constraints to an extended Kalman filter based approach to reduce the influence of poor initial parameter guesses.

These techniques for closed-loop identification purposes were addressed for open loop stable nonlinear systems. However, for industrial applications it is often necessary to account for process constraints. Model Predictive Control (MPC) strategy is usually considered for solving this kind of optimal control under constraints, which classically aims to drive the process state to a known target value (i.e. set-point or trajectory tracking). Recently, the field of economic MPC (EMPC) has emerged (see: (Rawlings et al., 2012), the recent special issue on this topic in the Journal of Process Control 24(8) and the references in (Ellis et al., 2014)). In contrary to classical MPC, EMPC aims to drive (most of the time) the process state in a particular time-varying state (that is unknown, in contrary to the MPC set-point) and which optimizes the defined economic cost function. In MPC and EMPC, some works are also dealing on stabilization issues (see for example (Huang et al., 2011; Zavala and Biegler, 2009)).

In this paper a closed loop on-line optimal identification approach for a class of dynamic systems is proposed, which optimizes an economic cost function and in the meantime maintains the closed loop asymptotically stable. Combining observer theory and nonlinear predictive control theory, the basis of the closed-loop OED for online identification approach proposed here was initially introduced in (Flila et al., 2008). In this initial work, the nonlinear model was linearized and the authors considered only the mono-variable case (a single input, a single measured state and a single unknown constant parameter) for stable nonlinear systems. In contrast to this previous work, the approach proposed here is developed for a general multi-variable case of open loop unstable or stable nonlinear systems, with input constraints and where the state may not be entirely measured. The novelty in the present paper is in the extension of the previous approach of (Qian et al., 2013) to guarantee local stability of the closed loop of nonlinear dynamic system by integrating a Lyapunov stability criterion (Calvet et al., 1989; Castillo et al., 2012) into the optimal control problem.

The paper is organized as follows. An outline of basic components and requirements needed for the proposed approach is presented in Section 2. Then in Section 3, the closed loop optimal identification approach for nonlinear dynamic model is developed which also stabilizes the system in a neighborhood of a steady state. This approach is illustrated in Section 4 through an example of a nonlinear open loop unstable dynamic system: a mechanical rolling delta wing. The obtained simulation results and analysis are given in Section 5.

\section{Problem statement}

\subsection{Class of systems considered}

The proposed approach is dedicated to processes that feature some dynamic behavior. Meanwhile, at least one output $y_{p}$ must be available as an on-line measure and the manipulation of at least one exogenous input $u$ must be possible on-line by a controller. Some constraints may be specified on the magnitude and velocity of the manipulated input ${ }^{1}$. Hence, this covers a very large number of potential applications.

Models considered here are nonlinear (or linear) in terms of state representation and/or in terms of model parameters. This dynamic multivariable model is described by ordinary differential equations

${ }^{1}$ Other constraints may be specified on the measured outputs or estimation of the process states (dealing with safety, set-point tracking within error bounds, production, ...). In order to handle the stability with the method detailed here, such constraints are not included. 
as follows:

$$
\left\{\begin{array}{l}
\dot{x}(t)=f(x(t), \theta, u(t)) \\
y(t)=h(x(t), \theta, u(t)),
\end{array}\right.
$$

${ }^{2}$ To simplify the notation in the following discrete formulations, $s(k)=s\left(t_{k}\right)$ (resp. $s(l)=s\left(t_{l}\right)$ ) represents the value of the signal $s$ at the current (resp. future) discrete time $t=k \times T_{s}$ (resp. $t=l \times T_{s}$ ), where $T_{s}$ is the constant sampling time and $k$ (resp. $l$ ) is a time index (i.e. an integer). For the input, a zero order hold is used between two consecutive sampling time instants. The various models are still formulated in a continuous framework and are solved numerically. Hence, sampled values may be taken at any discrete time. It is assumed that process data may also be sampled at the same rate. 
that all the model parameters are known, or that the state disturbance vector is bounded.

Based on this, the second control objective is to propose a method to solve online this constrained optimization problem (2-3) to find the optimal input $u(k)$ to apply at each time on the real process. The prediction of the output $y(l)$ in the cost function (2) depends also on the value of the parameter vector $\theta$ in the model (1). Here, since such parameters are unknown, they must be estimated online. In the field of parameter identification, one of the very common techniques in the engineering literature is based on the Kalman filter. In the following we recall main lines of this technique.

\subsubsection{Observer design for online identification}

This technique consists first in extending model (1) with its unknown constant parameters $\theta$ to be estimated (see for instance (Cox, 1964); (Nelson and Stear, 1976); (Ljung, 1979)):

$$
\left\{\begin{array}{l}
\dot{x}(t)=f(x(t), \theta, u(t)) \\
\dot{\theta}=0 \\
y(t)=h(x(t), \theta, u(t)) .
\end{array}\right.
$$

In the following, the augmented state vector is noted $x_{a}=\left[\begin{array}{ll}x & \theta\end{array}\right]^{T} \in \mathcal{R}^{n+q}$ and the vector function is $f_{a}=\left[\begin{array}{ll}f & 0\end{array}\right]^{T}$.

In the nonlinear case, different observer design techniques have been proposed such as: high gain observer (Gauthier et al., 1992), extended Kalman filter (EKF) (Besançon, 2007b) or adaptive-gain observer (Boizot et al., 2010; Nadri et al., 2013). The choice of the observer structure depends on the model structure and its observability property.

The nonlinear system (4) considered here may a priori admit inputs for which observability is not guaranteed (i.e. is not uniformly observable). Consequently, to design an observer for nonlinear systems requires the observability property which generally depends on the applied inputs (i.e. the sensitivity of the measurements with respect to the inputs). This addresses the well known important issue of persistency of excitation, which is still an open issue in the nonlinear case.

For state affine nonlinear systems, appropriate inputs have been characterized. In this case, observability corresponds to the notion of regularly persistent inputs which is clearly formulated using the Gramian of observability (Bornard et al. (1988)). We can find also some earlier results on input conditions to guarantee a possible observer design, depending on the system structure in (Besançon (2007c); Dufour et al. (2012)). However, the design of such inputs is still difficult and and in practice done heuristically.

Under observability conditions, a global observer can be designed for the augmented system (4):

Definition 1: A global observer based on the augmented model (4) can be given by a dynamical system as:

$$
\begin{cases}\dot{\hat{x}}_{a}(t) & =f_{a}\left(\hat{x}_{a}(t), u(t)\right)+g_{a}\left(t, h\left(\hat{x}_{a}(t), u(t)\right)-y_{p}(t)\right) \\ \text { with: } & g_{a}(t, 0)=0\end{cases}
$$

where $g_{a}$ is a function (of the output estimation error) to be designed and $y_{p}$ is the process output vector (on-line measures) such that

i) if $\hat{x}_{a}(0)=x_{a}(0)$, then $\hat{x}_{a}(t)=x_{a}(t), \quad \forall t \geq 0$;

ii) if $\forall x_{a}(0), \forall \hat{x}_{a}(0)$, then $\lim _{t \rightarrow+\infty}\left\|\hat{x}_{a}(t)-x_{a}(t)\right\|=0$.

The estimation problem consists in determining a gain structure $g_{a}$ and tuning the observer parameters such that the estimation error $e(t)=x_{a}(t)-\hat{x}_{a}(t)$ converges asymptotically to zero. Note that this convergence is also related to appropriate input excitation. 


\subsubsection{Problem considered}

From (5), the observer design problem may be turned into an optimization problem in the sense that we have to find an optimal input excitation $u$ (corresponding to the observability of the system) which guarantees the convergence of the estimation error.

Based on model (1) with unknown constant parameters $\theta$, a new optimal approach is proposed to guarantee the observability of system (4) through an optimal persistent input excitation calculated on-line.As a first step, an initial cost function is designed based on the sensitivity of the measurements with respect to the inputs to guarantee the persistence of the input. Then, the problem of closed-loop stability is considered. To guarantee this property, a second cost function is introduced into the optimization problem.

Remark 1: - Note that the proposed approach can be also relevant in the case of uniformly observable systems in the sense that the designed optimal input should improve the robustness of the observer with respect to measurement noise and model uncertainties.

- For a simple illustration of the approach, we assume in what follows that all the states are measured. However, in general the method does not require all states to be measured.

\section{A closed loop optimal identification approach}

\subsection{Structure for coupled control and online parameter identification}

In this paper, the developed general framework combines a closed loop OED with an observer for a nonlinear dynamic system, which can be open loop unstable. Based on the chosen model structure, an observer is designed for the augmented system.

Then, a sensitivity model is developed to capture the dynamics of the sensitivities of the state vector to the unknown model parameters. Finally, the outputs of the four components (a process, a model, an observer and a sensitivity model) are fed back into the optimal control problem which is solved by the EMPC strategy taking into account the stability analysis.

Fig. 1 shows how the components needed in the proposed closed loop identification algorithm are combined. In the following, the remaining components for the proposed closed loop optimal identification approach are presented, leading to the final optimal control problem.

\subsection{Sensitivity model}

The sensitivity model is explicitly deduced from model (1). Sensitivity analysis tells us how the unknown parameter vector $\theta$ affects the model state $x$ and the model output $y$.

Using the definition of the sensitivity function $(\cdot)_{\theta}=\frac{\partial(\cdot)}{\partial \theta}$ of a variable $(\cdot)$ with respect to the parameters $\theta$, and the dynamical model (1), one gets the sensitivity model:

$$
\left\{\begin{array}{l}
\dot{x}_{\theta}(t)=\frac{\partial f}{\partial x}(x(t), \theta, u(t)) x_{\theta}+\frac{\partial f}{\partial \theta}(x(t), \theta, u(t)) \\
y_{\theta}(t)=\frac{\partial h}{\partial x}(x(t), \theta, u(t)) x_{\theta}+\frac{\partial h}{\partial \theta}(x(t), \theta, u(t))
\end{array}\right.
$$

where $x_{\theta} \in R^{n \times q}$ and $y_{\theta} \in R^{p \times q}$ are the matrices of sensitivities of the states and the outputs, respectively, with respect to the parameters.

\subsection{Prediction model}

Based on model (1), the prediction model (7) aims to predict, at the current time $k$ and over the prediction horizon $N_{p}$, the future model output $y$. It is based on the current estimation $\hat{x}(k)$ of the 


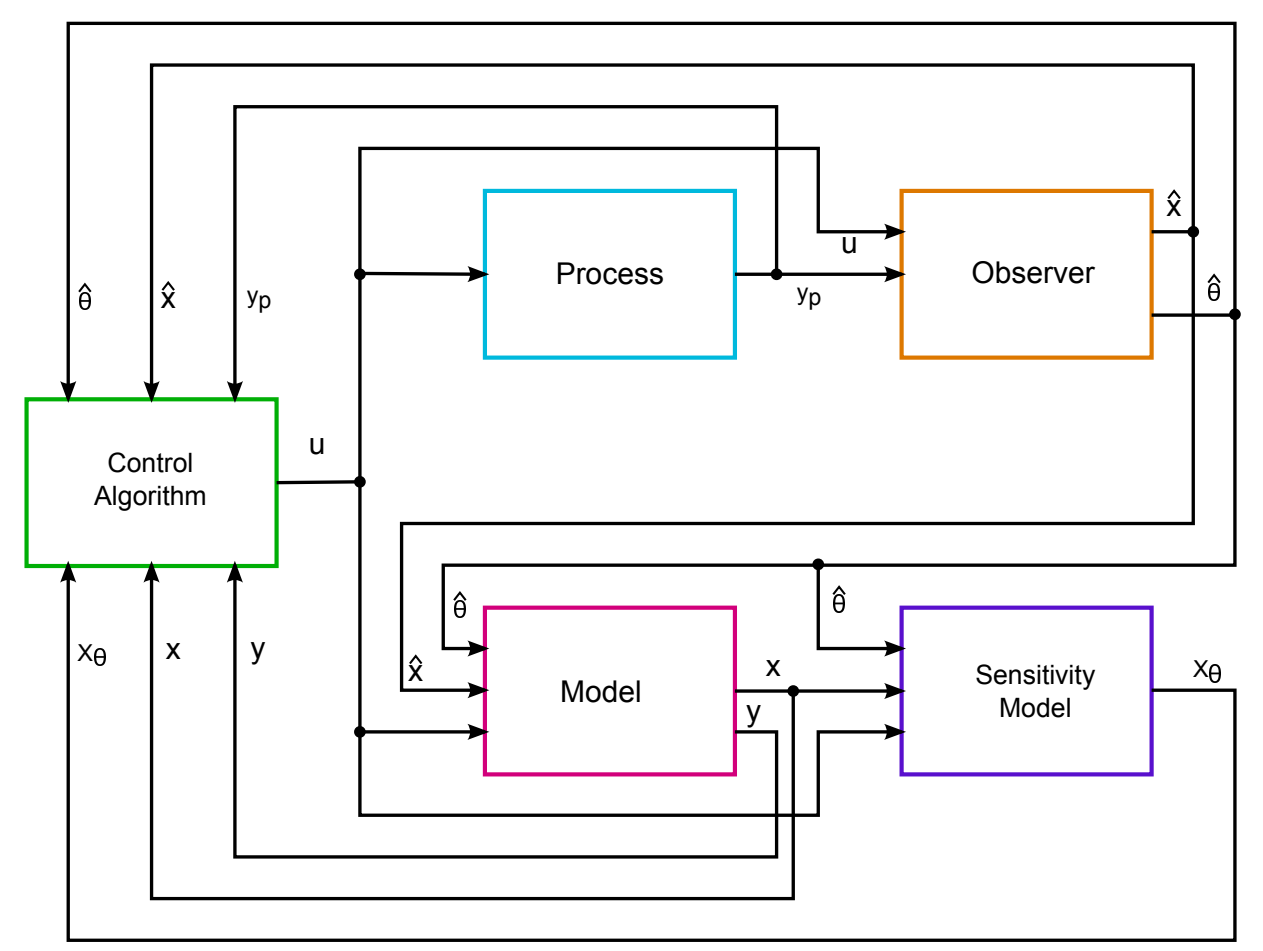

Figure 1. Closed loop control structure for on-line identification.

process state and the current estimation $\hat{\theta}(k)$ of the unknown constant parameters given by the observer (5):

$$
\left\{\begin{array}{rll}
\dot{x}(t) & =f(x(t), \hat{\theta}(k), u(t)) \forall t \in] t_{k}, & \left.t_{k+N_{p}}\right] \\
y(t) & =h(x(t), \hat{\theta}(k), u(t)) \forall t \in] t_{k}, & \left.t_{k+N_{p}}\right] \\
x\left(t_{k}\right) & =\hat{x}(k) .
\end{array}\right.
$$

\subsection{Prediction sensitivity model}

Based on the sensitivity model (6), the prediction sensitivity model (8) aims to predict, at the current time $k$ and over the prediction horizon $N_{p}$, the future sensitivity $y_{\theta}$ of the output of the prediction model (7) with respect to the current estimation $\hat{\theta}(k)$ of the unknown constant parameters:

$$
\left\{\begin{array}{rll}
\dot{x}_{\theta}(t) & =\frac{\partial f}{\partial x}(x(t), \hat{\theta}(k), u(t)) x_{\theta}(t)+\frac{\partial f}{\partial \theta}(x(t), \hat{\theta}(k), u(t)), & \left.\forall t \in] t_{k}, \quad t_{k+N_{p}}\right] \\
y_{\theta}(t) & =\frac{\partial h}{\partial x}(x(t), \hat{\theta}(k), u(t)) x_{\theta}(t)+\frac{\partial h}{\partial \theta}(x(t), \hat{\theta}(k), u(t)), & \left.\forall t \in] t_{k} \quad t_{k+N_{p}}\right] \\
x_{\theta}\left(t_{k}\right) & = \begin{cases}x_{\theta}(k \mid k-1), \quad \text { for } t_{k}>0 \\
0, \quad \text { for } t_{k}=0,\end{cases}
\end{array}\right.
$$

where $x_{\theta}(k \mid k-1)$ is the one step ahead solution of (8) at the time $k-1$.

Physical values involved in these sensitivities have usually different scales and units. So, in order to re-scale the effects of the different parameters on the different outputs of the model, each 
sensitivity is normalized with the relative-sensitivity function:

$$
\left\{\begin{array}{l}
\bar{x}_{i} \theta_{j}=\frac{\hat{\theta}_{j}(k)}{x_{i}} x_{i} \theta_{j} ; \quad i=1, \cdots, n ; j=1, \cdots, q \\
\bar{y}_{i} \theta_{j}=\frac{\hat{\theta}_{j}(k)}{y_{i}} y_{i} \theta_{j} ; \quad i=1, \cdots, p ; j=1, \cdots, q .
\end{array}\right.
$$

\subsection{Sensitivity criterion}

In the proposed approach, one of the objectives is to maximize the future information content of the experiment which must be described by a sensitivity criterion. First, one defines a sensitivity matrix $\bar{y}_{\theta}(l)$, which at the current instant $k$ gives the prediction at a future time $l>k$ of the normalized output sensitivity matrix $\bar{y}_{\theta}$ as

$$
\bar{y}_{\theta}(l)=\left[\begin{array}{llll}
\bar{y}_{1 \theta_{1}}(l) & \bar{y}_{1 \theta_{2}}(l) & \ldots & \bar{y}_{1 \theta_{q}}(l) \\
\bar{y}_{2 \theta_{1}}(l) & \ddots & & \vdots \\
\vdots & & \ddots & \vdots \\
\bar{y}_{p \theta_{1}}(l) & \ldots & \ldots & \bar{y}_{p \theta_{q}}(l)
\end{array}\right] .
$$

Using (10), we can now define the FIM as

$$
M(l)=\left\|\bar{y}_{\theta}(l)\right\|^{2} .
$$

This matrix contains the information of the experiment, at the current time $k$, for a future time $l>k$. Then, the classical $E$-optimality criterion

$$
\left.\left.J_{\theta}(l)=\left|\frac{\lambda_{\min }(M(l))}{\lambda_{\max }(M(l))}\right|, l \in\right] k k+N_{p}\right],
$$

is defined to be used in a maximization problem within a EMPC framework to get an OED.

The criterion given by (12) is specific to the maximization of the information contained in the FIM. Consequently, it does not take into account the eventual problem of system instability and the resulting optimal control does not ensure closed-loop system stability. In the following, this maximization problem is modified to take also this stability objective into account.

\subsection{Lyapunov local stability criterion}

The study of closed loop stability for nonlinear systems often relies on a Lyapunov function. Concerning EMPC, in (Alangar et al., 2015), the decrease of the derivative of the Lyapunov function is constrained by the predictive control resolution. But this relies on the perfect knowledge of the model parameters. In the case of open loop unstable nonlinear dynamic systems with uncertain parameters, it is always difficult to formulate an optimal control problem which can guarantee closed loop stability. Following the nonlinear robust control design methodology (Başar et al. (1995)), solving this problem relies on finding a positive definite and proper smooth Lyapunov function satisfying the nonlinear Hamilton Jacobi Bellman equation, which can be difficult (or impossible) to solve. However, if we focus on the linear approximation of the nonlinear system, then this problem can be solved locally. 
Now, let us consider the following controlled uncertain nonlinear system:

$$
\dot{x}=F(x, u)+d(t), \quad x(0)=x_{0},
$$

where $x$ in $\mathcal{R}^{n}$ is the state vector, $u$ in $\mathcal{R}^{m}$ is the control input, and $F: \mathcal{R}^{n} \rightarrow \mathcal{R}^{n}$ is a $C^{1}$ function such that ${ }^{3} F(0,0)=0$ and $d$ in $C_{0}\left(\mathcal{R} ; \mathcal{R}^{n}\right)$ is an unknown external disturbance. The function $F$ being smooth, we can introduce the two matrices $(A(t), B(t))$ in $\mathcal{R}^{n \times n} \times \mathcal{R}^{n \times m}$ with $A(t)=\left.\frac{\partial F}{\partial x}\right|_{(x, u)}$ and $B(t)=\left.\frac{\partial F}{\partial u}\right|_{(x, u)}$ to describe the first order approximation of system (13) at $(x(t), u(t))$. All along this paper, it is assumed that the system (13) satisfies the following assumption:

Assumption 2 (First order controllability:): The pair of matrices $(A(t), B(t))$ is controllable $\forall t$.

Based on Assumption 2, we focus on the design of an optimal local controller for the linear time varying system:

$$
\dot{x}(t)=A(t) x(t)+B(t) u(t)+g(x, t)
$$

where $A($.$) and B($.$) are time varying state matrices given by (13) . g(x, t)$ is a locally Lipschitz continuous function nearby $x=0$ such that $g(0, t)=0, \forall t>0$, and which can be considered as a "disturbance" of the system:

- if $g(x, t)=0$, the system (14) is non-autonomous linear.

- if $\lim _{\|x\| \rightarrow 0, x \neq 0} \frac{g(x, t)}{\|x\|}=0$ uniformly with respect to $t$, the system (14) is non-autonomous quasilinear system (Calvet et al., 1989; Willems, 1970).

In the context of MPC technique, at time $k$, an update of the model parameters $\hat{\theta}(k)$ is done and the time varying matrices $A(t)$ and $B(t)$ of the linearized system (14) are updated $(A(k), B(k))$ and considered constant over the prediction horizon $N_{p}$. Consequently, at time $k$, the Hamilton Jacobi Bellman equality is an algebraic equation which is easy to solve :

$$
P(k) A(k)+A(k)^{T} P(k)-P(k) B(k) R^{-1} B(k)^{T} P(k)+Q=0,
$$

where the unique solution $P(k)$ is a positive definite matrix in $\mathcal{R}^{n \times n}$, with both $Q$ and $R$ are positive definite matrices.

Now, let us assume that $(A(k), B(k))$ is stabilizable and $\left(A(k), Q^{\frac{1}{2}}\right)$ is detectable, then $V(x)=x^{T} P(k) x$ is a Lyapunov function for system (14).

Based on this, the optimal input $u$ minimizing the cost :

$$
J(u)=x^{T}(t) P(k) x(t)
$$

stabilizes the solution of system (14) to the origin.

Then, based on the prediction of the model states $x(l)$, a stability criterion is established as a quadratic Lyapunov function as follows:

$$
\left.\left.J_{L}(l)=x^{T}(l) P(k) x(l), l \in\right] k k+N_{p}\right] .
$$

\footnotetext{
${ }^{3}$ As usual, the same development can be done for any pair $(x, u) \neq(0,0)$ that represents a steady state in (13).
} 
By construction, this criterion $J_{L}$ is therefore a positive definite and decreasing function. Consequently, the input sequence $\tilde{u}(l \mid k)$ minimizing $J_{L}$ allows to stabilize locally asymptotically the system (13) in closed loop.

In the following, the stability criterion $J_{L}(17)$ based on $P(k)$ has to be combined with the sensitivity criterion $J_{\theta}(12)$ to get the final criterion to maximize such that the EMPC can also handle local stability. Otherwise, since that linearization is done around the augmented state vector, robust stabilization particularly depends on the quality of convergence of the estimation error. However, based on the update of the linearization at every time $k$ and thanks to the optimization algorithm which allows to increase the robustness of the observer to the measurements noise an uncertainties, the local stability in closed loop is handled.

\subsection{Final optimal control problem formulation}

In (Qian et al., 2013) a first approach was designed based on an EMPC, where the cost function aimed only to maximize the FIM of the experiment, while some fictitious output constraints were designed by trial and error to stabilize the process in closed loop. The main drawback is that design and tuning of output constraints is not easy. Compared to this result, in the present work, instead of using fictitious output constraints, a dual cost function is formulated in an EMPC which combines two parts in a constrained maximization problem: one part is, as previously (Qian et al., 2013), a sensitivity criterion (12) to improve parameter estimation, and the second part (the new part) is based on a Lyapunov function (17) deduced from Lemma 1 to locally asymptotically stabilize the process in closed loop. Indeed, on one hand, if only the maximization of the FIM is considered, the closed loop system may become unstable. On the other hand, if only the closed loop stability of the system is considered, the information content in the experience may not be rich enough to guarantee accuracy of the parameter estimation step. Similarly to the discussion on the theorem 3 in (Angeli et al., 2012), the solution adopted here is to find the optimal control which leads to a balance between parameter estimation and the stability of the system in closed loop. Hence, one formulates the final optimal identification problem as follows:

$$
\left\{\begin{array}{l}
\tilde{u}^{*}(l \mid k)=\arg \max _{\tilde{u}(l \mid k)} J \\
\tilde{u}(l \mid k)=\left\{u(k) \cdots u(l) \cdots u\left(k+N_{p}\right)\right\}, l \in\left[k k+N_{p}\right] \\
J=\frac{1}{N_{p}} \sum_{l=k+1}^{k+N_{p}}\left(\beta \frac{J_{\theta}(l)}{w_{\theta}}-(1-\beta) \frac{J_{L}(l)}{w_{L}}\right) \\
\text { under the input constraints, }(\forall k>0): \\
\left\{\begin{array}{l}
u_{\min } \leqslant u(k) \leqslant u_{\max } \\
\Delta u_{\min } \leqslant \frac{u(k)-u(k-1)}{T_{s}} \leqslant \Delta u_{\max }
\end{array}\right. \\
\text { based on the observer }(5), \\
\text { the prediction model }(7), \text { the sensitivity model (8), } \\
\text { the sensitivity criterion (12) and the stability criterion (17). }
\end{array}\right.
$$

Remark 2: Note that this formulation does not aim to solve a set-point regulation problem but the OED. Therefore, only the input is constrained. Indeed, adding tight state constraints can be penalizing for such a design of persistent excitation and handling such constraints requires more knowledge on the system. Meanwhile, to ensure closed loop stability, the Lyapunov term (17) replaces (in the sense of closed loop stability) the previous fictitious constraints on the output (Qian et al., 2013).

Here, $J$ is the final optimization criterion to maximize, $\beta$ is the real weighting tuning parameter in $[0,1]$ used to mix the two competitive parts involved in the cost function: the sensitivity criterion 
$J_{\theta}$ and the Lyapunov stability criterion $J_{L}{ }^{4}$. Since these two criteria may have different scales, two weights $w_{\theta}$ and $w_{L}$ are defined, to normalize $J_{\theta}$ and $J_{L}$ respectively in $J$. Hence, the pure sensitivity maximization is one of the two extremes (i.e. $\beta=1$ ) where the outputs contain the maximum of information in all the unknown model parameters but closed loop stability is not ensured. On the other extreme (i.e. $\beta=0$ ), the pure stabilization problem is shown to have a well defined Lyapunov function. In the meantime, the information content in the outputs might be not rich enough, which may render the accurate estimation of all parameters of the model impossible. This will be illustrated with the tuning of $\beta$ in the case study in Section 5. Hence an optimal control $\tilde{u}^{*}(l \mid k)$ is obtained that in a unique experience maximizes the content of information fed into the observer used for the online parameter estimation, and meanwhile locally stabilize the system and solve the disturbance attenuation in closed loop.

Moreover, in contrary to the case studied in (Angeli et al., 2012), the weighting matrix $P$ involved in the stability criteria $J_{L}$ is not constant but is adapted online according to the estimation of the model parameters.

Note also that, we do not need here to specify a weighting matrix to penalize the deviation from the control input $u$ away from 0 (the steady input).

The robustness of the proposed approach to measurements noise and model uncertainties relies on the choice of the sensitivity cost function (12). However, to preserve this performance, the choice of the parameter $\beta$ is important and should be done based on prior knowledge on the real system.

\subsection{Implementation of the algorithm}

Based on the nonlinear constrained optimal problem (18) to be solved online, the implementation of the EMPC strategy can be summarized as follows, at the current instant $k$ :

- Step 1: update the input/output measures and apply at the process input the first component of $\tilde{u}^{*}(l \mid k-1)$ (with a zero order hold).

- Step 2: integrate the observer (5) to get an estimate of the state $\hat{x}(k)$ and the unknown constant parameters $\hat{\theta}(k)$, based on the current input and output measures.

- Step 3: solve online the nonlinear constrained optimization problem (18) to get the optimal control sequence $\tilde{u}^{*}(l \mid k)$. This requires integrating the prediction model (7) and the prediction sensitivity model (8) over the prediction horizon for all control sequence guesses $\tilde{u}(l \mid k)$. It is based on the current input and output measures, the unknown parameter estimations $\hat{\theta}(k)$ (considered as constant over the prediction horizon) and the state estimations $\hat{x}(k)$

- Step 4: The first element $u^{*}(k \mid k)$ of the optimal control sequence $\tilde{u}^{*}(l \mid k)$ is applied at the next sampling time $k+1$ (via a zero order blocker).

At the next discrete time the whole procedure is repeated. Finally, this optimal control problem is solved to estimate the unknown constant parameters, excite the system and stabilize the closed loop.

This algorithm has been implemented in Matlab and under the name ODOE4OPE software $^{5}$. The models are solved with the Matlab ODE solvers. The local solution of the constrained optimization problem is obtained by the Matlab fmincon routine.

It has to be noticed that this method may lead to a computational burden, especially for large size systems with relatively short sampling times. Indeed, the search for the optimal $\tilde{u}^{*}(l \mid k)$ at each time $k$ may be time consuming since the prediction model, the prediction sensitivity model, the minimum and maximum eigenvalues of the predicted FIM must be computed repeatedly for each guess of $\tilde{u}(l \mid k)$.

${ }^{4}$ As discussed previously, since it is a Lyapunov function and since the cost function $J$ has to be maximized, one adds a minus sign before $J_{L}$

${ }^{5}$ More information on odoe4ope.univ-lyon1.fr 


\section{A rolling delta wing: the step by step procedure}

In this section, the developed closed loop on-line parameter identification approach is illustrated step by step for an open loop unstable dynamic system: a mechanical rolling delta wing. This application has been studied in (Jain et al., 2005) and in (Qian et al., 2013), whose results will be compared to the simulation results obtained with the approach presented here.

\subsection{Step 1: Model, open loop analysis and recent works}

The nonlinear model features a single dimensionless input $u(t)$, a two dimensionless component state vector $x(t)$ and the five unknown dimensionless constant parameter vector $\theta$ (Jain et al., 2005):

$$
\begin{cases}\dot{x}_{1}(t) & =x_{2}(t) \\ \dot{x}_{2}(t) & =\alpha_{1} \theta_{1} x_{1}(t)+\left(\alpha_{1} \theta_{2}-\alpha_{2}\right) x_{2}(t) \ldots \\ & \ldots+\alpha_{1} \theta_{3} x_{1}^{3}(t)+\alpha_{1} \theta_{4} x_{1}^{2} x_{2}(t)+\alpha_{1} \theta_{5} x_{1} x_{2}^{2}(t)+\alpha_{3} u(t)\end{cases}
$$

where $t$ is the dimensionless time and $\alpha$ is the known constant parameter vector. The dimensionless numerical values of constant known parameters and target values of the unknown parameters obtained from (Nayfeh et al., 1989) are listed in the table 1. To underline the open loop instability

Table 1. Constant parameters: unknown values (top) and known values (bottom) (Nayfeh et al., 1989).

\begin{tabular}{cccccc}
\hline index & 1 & 2 & 3 & 4 & 5 \\
\hline$\theta$ & -0.05686 & 0.03254 & 0.07334 & -0.3597 & 1.4681 \\
\hline
\end{tabular}

\begin{tabular}{cccc}
\hline index & 1 & 2 & 3 \\
\hline$\alpha$ & 0.354 & 0.001 & 1 \\
\hline
\end{tabular}

of the system, the nonlinear system (19) is first linearized around the steady state $\left(u^{0}, x^{0}\right)$, with $u^{0} \in\left[\begin{array}{ll}u_{\min } & u_{\max }\end{array}\right]=\left[\begin{array}{ll}-0.01 & 0.01\end{array}\right]$ (which is in the following the domain of interest for this system).

Figure 2 shows each pair of steady states $\left[\begin{array}{ll}x_{1}^{0} & x_{2}^{0}\end{array}\right]$ and the real part of each eigenvalue of the linearized system for the corresponding input $u^{0}$. The real parts of two eigenvalues are always positive, which presents the instability of the delta wing model in open loop, and therefore the need to design a stabilizing controller.

In previous work (Jain et al., 2005), a closed loop identification of the five unknown model parameters is discussed. A feedback linearizing control is used such that the closed-loop behavior matches with a specified second order linear reference model response with damped sinusoidal input reference. Both designs of this model and its input reference are not really discussed in a general framework. With their approach, the convergence of the two parameter estimations $\left(\theta_{1}\right.$ and $\left.\theta_{2}\right)$ to their targets is reported, while the convergence of the three remaining parameter estimations $\left(\theta_{3}\right.$, $\theta_{4}$ and $\left.\theta_{5}\right)$ to their target is not possible.

In 2013 (Qian et al., 2013), the sensitivity criterion E-optimality is used as the cost function of the optimal control problem. To stabilize the system in a prescribed region, two fictitious output constraints were imposed and designed by trial and error method. The convergence of the five 

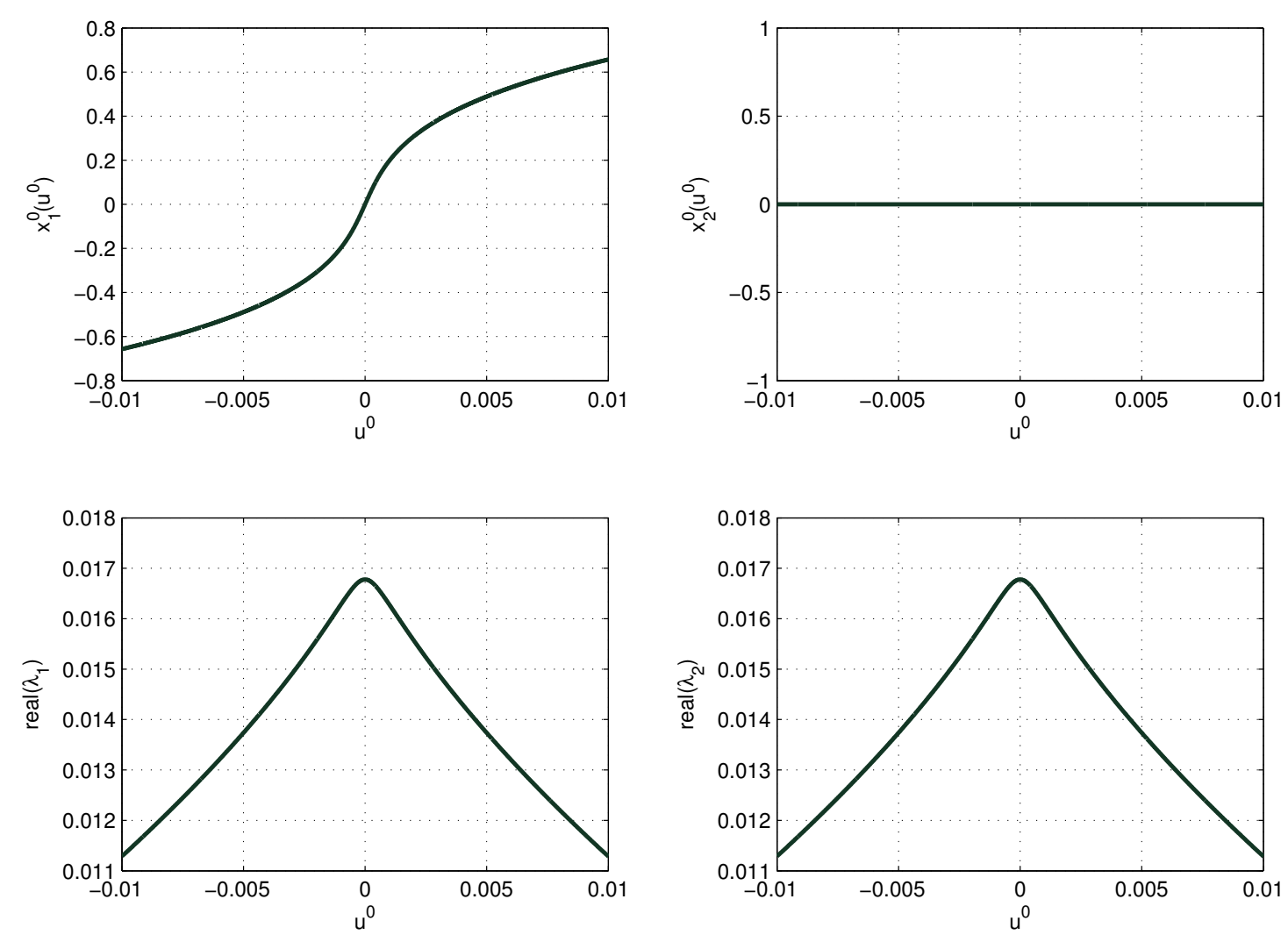

Figure 2. Linearized system of (19), for $u^{0}$ in [-0.01 0.01]: Steady state $x_{1}^{0}$ (top left) and $x_{2}^{0}$ (top right), real part of each eigenvalue (bottom left and right).

unknown constant parameter estimations to their target is obtained with good accuracy and the system is stable in closed loop.

\subsection{Step 2: Observer design}

Similarly to the work of (Jain et al., 2005), both the states here are on-line measured. Hence, the purpose of the observer to be designed is to estimate on-line the vector of unknown parameters $\theta_{i}(i=1, \cdots, 5)$. To do so, according to the augmented model (4), system (19) is extended by the vector $\dot{\theta}=0$. Consequently, the obtained augmented model is a state affine system up to nonlinear output injection in the following form

$$
\left\{\begin{array}{l}
\dot{x}_{a}(t)=A_{a}(y(t)) x_{a}(t)+B_{a}(u(t)) \\
y(t)=C_{a} x_{a}(t)
\end{array}\right.
$$


where $x_{a}(t)=\left[\begin{array}{c}x_{1}(t) \\ x_{2}(t) \\ \theta_{1} \\ \theta_{2} \\ \theta_{3} \\ \theta_{4} \\ \theta_{5}\end{array}\right] ; B_{a}\left(u(t)=\left[\begin{array}{c}0 \\ -\alpha_{3} u(t) \\ 0 \\ 0 \\ 0 \\ 0 \\ 0\end{array}\right] ; C_{a}^{T}=\left[\begin{array}{c}I_{2 \times 2} \\ 0_{5 \times 2}\end{array}\right] ;\right.$
with $A_{a}(y(t))=\left[\begin{array}{cc}0_{2 \times 1} & A(y(t)) \\ 0_{5 \times 1} & 0_{5 \times 6}\end{array}\right] ; A^{T}(y(t))=\left[\begin{array}{cc}1 & -\alpha_{2} \\ 0 & \alpha_{1} y_{1}(t) \\ 0 & \alpha_{1} y_{2}(t) \\ 0 & \alpha_{1} y_{1}^{3}(t) \\ 0 & \alpha_{1} y_{1}^{2}(t) y_{2}(t) \\ 0 & \alpha_{1} y_{1}(t) y_{2}^{2}(t)\end{array}\right]$,

$I_{2 \times 2}$ is the $2 \times 2$ identity matrix, $0_{a \times b}$ is the $a \times b$ matrix of zeros.

Then, a high gain observer based on the augmented state $x_{a}(t)$ can be designed as follows (see (Hammouri and Morales, 1990) and (Besançon, 2007a) for more details). For any $x_{a}(0)$, the system (20) admits an exponential observer of the form

$$
\left\{\begin{array}{l}
\dot{\hat{x}}_{a}(t)=A_{a}(y(t)) \hat{x}_{a}(t)+B_{a}(u(t))-\Gamma S_{\mu}(t)^{-1} C_{a}^{T}\left(C_{a} \hat{x}_{a}(t)-y_{p}(t)\right) \\
\dot{S}_{\mu}(t)=-\mu S_{\mu}(t)-A_{a}(y(t))^{T} S_{\mu}(t)-S_{\mu}(t) A_{a}(y(t))+C_{a}^{T} \Gamma C_{a}
\end{array}\right.
$$

where $S_{\mu}$ is a $7 \times 7$ symmetric positive definite matrix, the positive constant $\mu>0$ and $\Gamma>1$ are the observer tuning parameters.

\subsection{Step 3: Sensitivity model}

Based on the model (19), the sensitivity model (6) simply becomes:

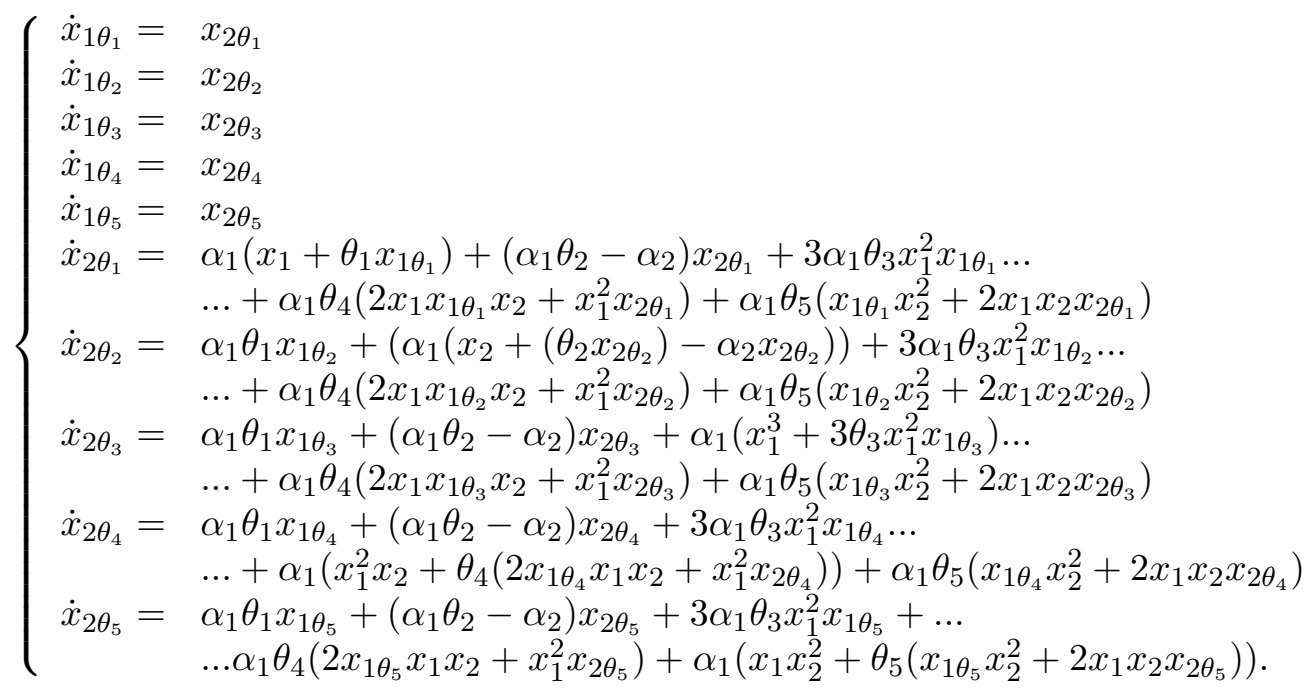

\subsection{Step 4: Control design}

As described in section 3 , the outputs of the process $y_{p}$, the state $x$ of the model (19), the state $\hat{x}_{a}$ of the observer (20) and the state $x_{\theta}$ of the sensitivity model (22) are fed back into the control law. In (18), the cost function is built from two parts: the sensitivity criterion and the Lyapunov stability criterion. The outputs of the sensitivity model are normalized using the relative-sensitivity 
function (9) in order to establish the sensitivity criterion (12). At the same time, a Lyapunov stability analysis is also needed to stabilize the system in closed loop. For determining the Lyapunov function (17), let us first consider system (19) as a quasi-linear system (14) in which

$$
\left\{\begin{array}{l}
\text { At the current time } k, \text { over the prediction horizon } \\
A(k)=\left[\begin{array}{cc}
0 & 1 \\
\alpha_{1} \hat{\theta}_{1}(k) & \alpha_{1} \hat{\theta}_{2}(k)-\alpha_{2}
\end{array}\right] \\
B(k)=\left[\begin{array}{c}
0 \\
\alpha_{3}
\end{array}\right] \\
g(x, k)=\left[\begin{array}{c}
\alpha_{1} \hat{\theta}_{3}(k) \hat{x}_{1}^{3}(k)+\alpha_{1} \hat{\theta}_{4}(k) \hat{x}_{1}^{2}(k) \hat{x}_{2}(k) \ldots \\
\ldots+\alpha_{1} \hat{\theta}_{5}(k) \hat{x}_{1}(k) \hat{x}_{2}^{2}(k)
\end{array}\right],
\end{array}\right.
$$

where the function vector $g(x, k)$ is assumed to be a "small disturbance" of the system and respects the condition of non-autonomous quasi-linear systems (Calvet et al., 1989). Then based on the constant matrices $A(k)$ and $B(k)$ over the prediction horizon, one computes the positive semidefinite matrix $P(k)$ by solving the algebraic Riccati equation (15). Finally, using the computed $P(k)$ and the prediction of model states $x(l)$, one gets the Lyapunov function (17). Effects of tuning the weighting value $\beta$ are discussed in the following section.

\section{A rolling delta wing: numerical simulation results}

\section{$5.1 \quad$ Numerical conditions}

All simulation runs are performed under the following conditions where all values are dimensionless ${ }^{6}$

$$
\left\{\begin{array}{l}
\text { input constraints: }-0.01 \leq u(k) \leq 0.01 \\
\text { prediction horizon: } N_{p}=5 \\
\text { sampling time: } T_{s}=1 \\
\text { observer tuning parameters: } \mu=0.03, S_{\mu}=I_{7 \times 7} \text { and } \Gamma=2 \\
\text { parameters in Riccati equation: } Q=0.01 I_{2 \times 2} \text { and } R=I \\
\text { weights in the cost function: } w_{\theta}=10^{-14} \text { and } w_{L}=1
\end{array}\right.
$$

Initial estimation errors for $\theta_{i}$ are listed in table 2: in order to see the robustness of the approach, large initial errors in the estimation of $\theta_{i}$ are introduced, including even sign errors.

Table 2. $\theta$ : initial estimation errors.

\begin{tabular}{cccccc}
\hline$\theta$ & 1 & 2 & 3 & 4 & 5 \\
\hline Initial error (\%) & 80 & -200 & 200 & 80 & -200 \\
\hline
\end{tabular}

The simulation runs are performed under the ODOE4OPE software ${ }^{7}$ based on Matlab.

\section{$5.2 \quad$ Influence of the weighting value $\beta$}

As discussed before, the weighting value $\beta$, ranging between 0 an 1, balances the dual cost function to obtain the convergence of the five unknown parameter estimation and also stabilizes the system in closed loop. In order to study the effects of the weighting value $\beta$, a series of simulations is presented

${ }^{6}$ The time, the input and the states are also dimensionless.

${ }^{7}$ To use this software, please visit http://odoe4ope.univ-lyon1.fr 
with different values of $\beta$ to compare the convergence of the unknown parameter estimations to their targets and the closed loop stability. Moreover, to see the impact of noise measurement, two cases are studied to show the robustness of the proposed closed loop on-line optimal identification approach defined in this paper:

- Case 1: without noise.

- Case 2: with a 5\% Gaussian noise on the output measurements.

Table 3 presents the mean value (resp. the norm of the mean value) of the last 60 values of the estimation of the five parameters in the two cases. As it can be seen here, in the case without noise, for several weighting values $\beta$ (i.e. 0.3, 0.5, 0.6 and 0.7), and in spite of large initial error, the final estimation error of $\theta_{i}$ tends to zero (the maximal final estimation error is less than $9 \%$ (often less than $1 \%$ ) and the minimum is less than $1 \%$ ), which means the convergence of the five parameter estimations to their targets is achieved. With $\beta$ tuned between 0.2 and 0.8 in the case without noise, it is robust for both the parameter estimations and the closed loop stability. In the case with noise, table 3 shows that for $\beta=0.6$, the most accurate parameter estimations are obtained: all five final estimation errors are less than $3 \%$. The closer the weighting value $\beta$ approaches its limits 0 or 1 , the larger the parameter estimation errors are: Indeed, for $\beta=0$ (i.e., the cost function is only dealing with the stability) in the two cases, both input $u$ and model state $x$ tend as expected towards zero, but there is not enough information for parameter estimation: the estimation convergence for the two first components of the parameter vector is obtained, but the estimation for the other three other parameters is not possible (see table 3 ). For $\beta \geqslant 0.8$ in the case without noise or for $\beta \geqslant 0.7$ in the case with noise (i.e. maximizing sensitivity criterion is more important than stabilizing the system), the system response is completely divergent. In other words the system is unstable in closed loop, so parameter estimation is impossible.

Table 3. Influence of the tuning of $\beta$ : Final mean parameter estimation error (\%) for each parameters and for the whole parameter vector, and states stability.

\begin{tabular}{|c|c|c|c|c|c|c|c|c|}
\hline$\beta$ & 1 & 2 & $\begin{array}{c}\theta_{i} \\
3\end{array}$ & 4 & 5 & $\begin{array}{c}\text { Vector } \\
\theta\end{array}$ & $x_{1}$ & $x_{2}$ \\
\hline 0 without noise & -0.003 & 8 & 179 & 79 & -200 & 125 & stable & stable \\
\hline 0 with noise & 0.5 & -13 & 178 & 79 & -200 & 125 & stable & stable \\
\hline 0.3 without noise & 0.01 & 2 & 0.2 & -1 & -1 & 1 & stable & stable \\
\hline 0.3 with noise & 0.01 & 2 & 16 & 4 & -62 & 27 & stable & stable \\
\hline 0.5 without noise & -0.06 & 0.4 & -0.5 & 1 & 2 & 1 & stable & stable \\
\hline 0.5 with noise & 0.4 & 7 & 4 & 14 & -4 & 7 & stable & stable \\
\hline 0.6 without noise & -0.2 & 3 & 0.2 & 8 & -5 & 5 & stable & stable \\
\hline 0.6 with noise & -0.05 & -1 & -0.06 & -2 & 0.7 & 1 & stable & stable \\
\hline 0.7 without noise & 0.1 & -2 & 0.04 & -0.4 & 2 & 1 & stable & stable \\
\hline 0.7 with noise & -0.3 & 16 & 2 & 2 & -6 & 8 & unstable & unstable \\
\hline 0.8 without noise & 1 & 8 & 3 & 0.2 & -19 & 9 & unstable & unstable \\
\hline 0.8 with noise & 2 & -19 & 8 & 2 & -63 & 30 & unstable & unstable \\
\hline 1 without noise & 359 & -250 & 792 & 827 & 22 & 548 & unstable & unstable \\
\hline 1 with noise & 48 & 120 & 50 & -16 & -140 & 88 & unstable & unstable \\
\hline
\end{tabular}

The optimal tuning for $\beta$ is almost similar in the two cases: 0.5 for the case without noise, and 0.6 for the case with noise. This tuning is therefore robust with respect to the noise.

\subsection{Simulation results for $\beta=0.6$, with output noise}

Applying the proposed developed approach on the delta wing system, the results for the case of $\beta=0.6$ with output noise are presented here in more details. Figure 3 (a zoom is shown in Figure 4) presents the closed loop optimal control time behavior within input constraints which stabilizes 
the rolling delta wing behavior as shown in Figure 5. The first component $y_{p_{1}}$ of the output $y_{p}$ is stabilized in the region between -0.6 and 0.8 (which is larger than the output constraint set defined by trial and error in (Qian et al., 2013)), and the second component $y_{p_{2}}$ of the output $y_{p}$ is maintained between -0.1 and 0.1. In Figures 6 and 7, the five parameter targets and time evolution of the estimations are normalized (hence, 1 is the target): all unknown parameters are estimated. Meanwhile, the parameter $\theta_{1}$ reaches its target in $t=200$, the parameter $\theta_{2}$ reaches its target before $t=600$ (Figure 6), and the other three parameters converge to their targets around $t=500$ (Figure 7). Therefore, the optimal control stabilizes the system in closed loop and helps to estimate all five unknown parameters with high accuracy.

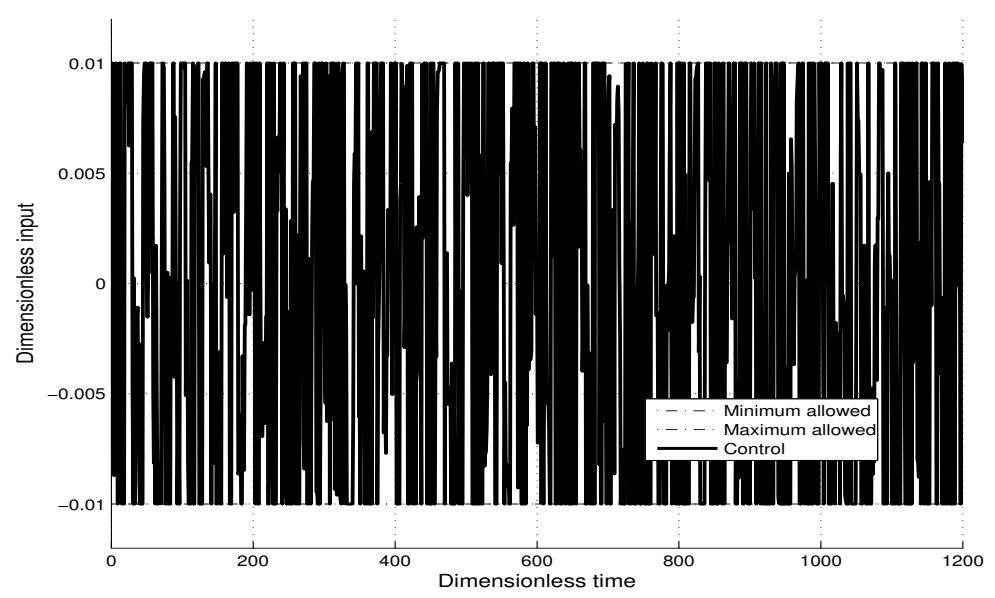

Figure 3. Closed loop optimal input for $\beta=0.6$ with output noise.

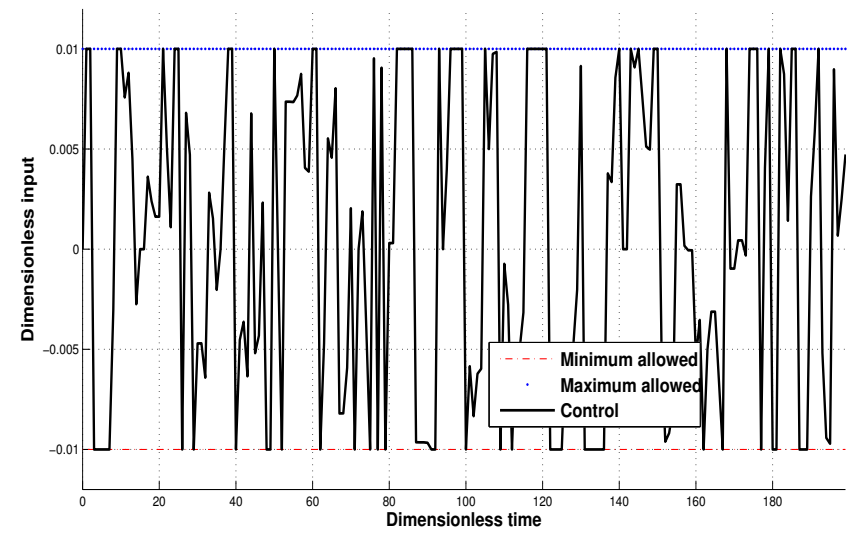

Figure 4. Closed loop optimal input for $\beta=0.6$ with output noise (zoom on $0 \leq t \leq 200$ ).

\section{Conclusion}

In this paper, a closed loop controller for on-line parameter identification was designed for a general class of nonlinear dynamic systems. An optimization problem was formulated based on a sensitivity criterion and a Lyapunov function. Indeed, based on the observer theory and the MPC strategy, a cost function was maximized online to get a trade-off between local stability and the observer robustness by tuning a unique controller parameter. Consequently, stability was guaranteed in 

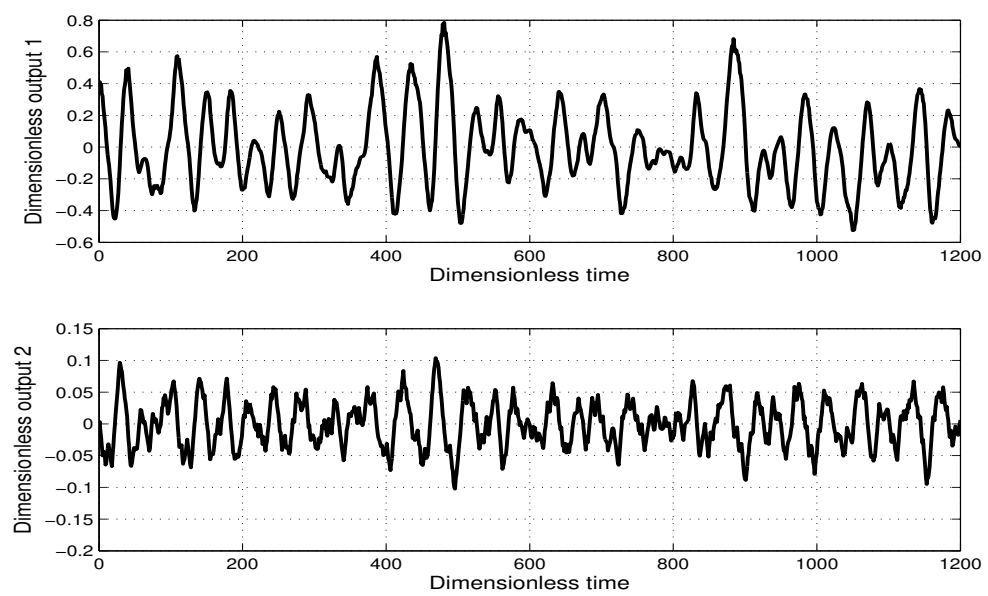

Figure 5. Closed loop outputs for $\beta=0.6$ (top: $y_{p 1}$, bottom: $y_{p 2}$ ) with output noise.
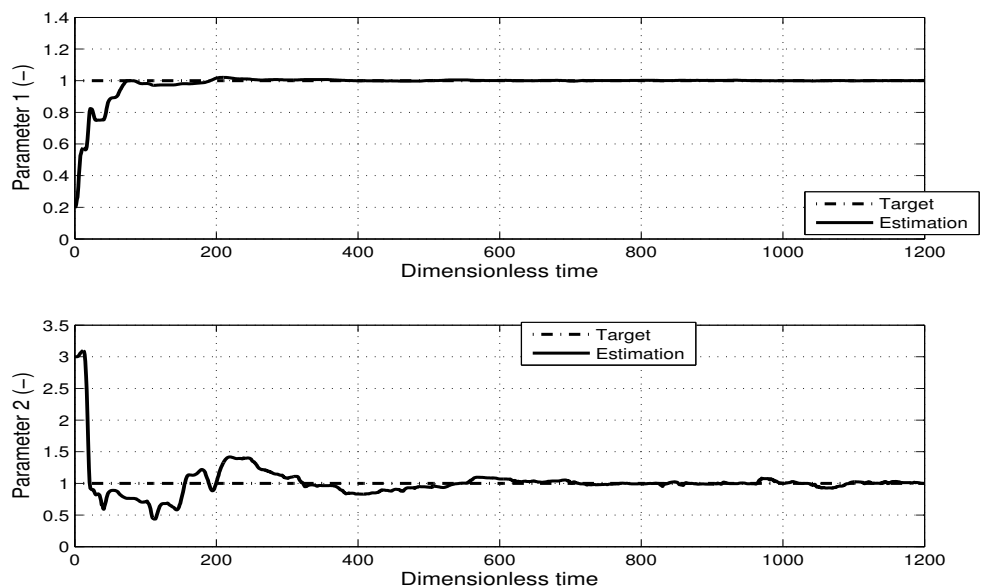

Figure 6. Closed loop estimation for $\beta=0.6$ (from the top to the bottom: $\theta_{1}$ and $\theta_{2}$ ) with output noise.
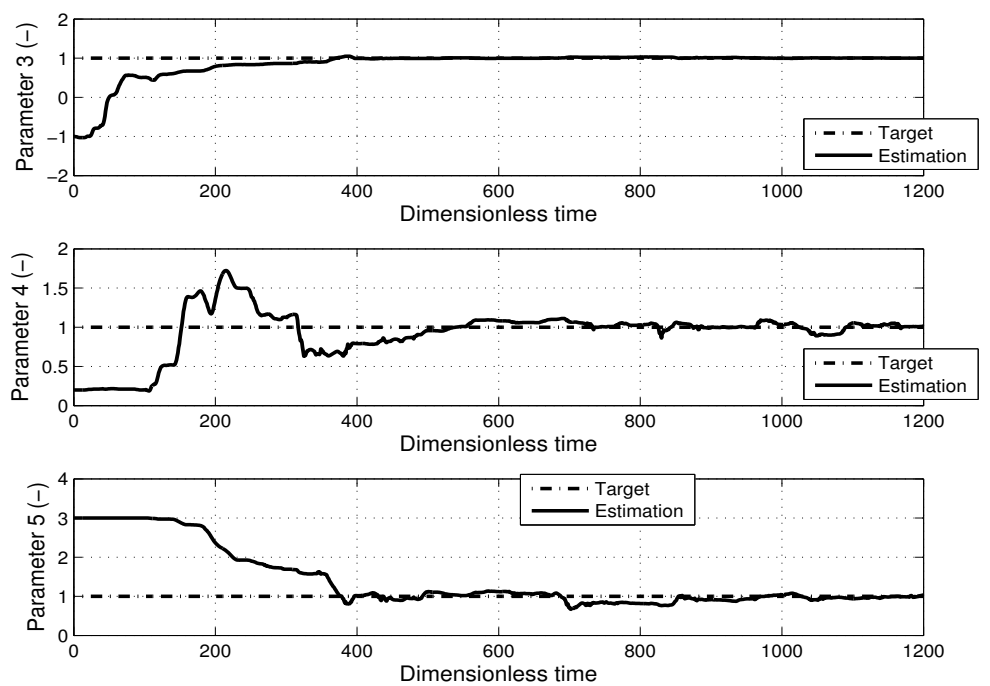

Figure 7. Closed loop estimation for $\beta=0.6$ (from the top to the bottom: $\theta_{3}, \theta_{4}$ and $\theta_{5}$ ) with output noise. 
closed loop by Lyapunov analysis (while previous results were based on a trial and error method) and the estimation error converged asymptotically to zero. This generic approach was illustrated step by step with simulations on an aircraft example (an open loop unstable mechanical delta wing system). In contrary to a previous work, the convergence of all five parameter estimations to their target and the stability of closed loop were shown with noisy output measurements.

As possible future works, it could be discussed how to add output constraints to keep also the system in a desired production area. It should then be considered how this competes with the stability criteria. Also, a formal proof of robustness needs to be addressed, to minimize the impact of the transient estimation error on the closed loop control objectives. Interval observers, as a tool to give the estimation bounds, may be considered.

\section{Funding}

This work was a part of the PhD thesis CIFRE 2011/0876 of J. Qian between the french company Acsystème and the french ministry of higher education and research, which is acknowledged for the funding support.

\section{References}

Alanqar, A., Ellis, M., \& Christofides, P.D. (2015). Economic model predictive control of nonlinear process systems using empirical models. AIChE Journal, 61(3), 816-830.

Angeli, D., Amrit, R., \& Rawlings, J.B. (2012). On average performance and stability of economic model predictive control. IEEE Transactions On Automatic Control, 57(7), 1615-1626.

Barz, T., Cárdenas, D.C.L., Arellano-Garcia, H., \& Wozny, G. (2012). Experimental evaluation of an approach to online redesign of experiments for parameter determination. AIChE Journal, 59(6), 1981-1995.

Başar, T. \& Bernhard, P. (1995). $H_{\infty}$-optimal control and related minimax design problems: a dynamic game approach. Birkhauser.

Besançon, G. (2007a). Identification of parametric models from experimental data. Lecture Notes in Control and Information Sciences, 363, Berlin Heidelberg:Springer-Verlag.

Besançon, G. (2007b). Nonlinear observers and application. Lecture Notes in Control and Information Sciences, 363, Berlin Heidelberg:Springer-Verlag.

Besançon, G. \& Ticlea, A. (2007). An immersion-based observer design for rank-observable nonlinear systems. IEEE Transactions on Automatic Control, 52(1), 83-88.

Blanc, D., Dufour, P., Toure, Y., \& Laurent, P. (2003). On nonlinear distributed parameter model predictive control strategy: on-line calculation time reduction and application to an experimental drying process. Computers and Chemical Engineering, 27, 1533-1542.

Boizot, N., Busvelle, E., \& Gauthier, J.-P. (2010). An adaptive high-gain observer for nonlinear systems. Automatica, 46(9), 1483-1488.

Bornard, G., Couenne, N. \& Celle, F. (1988). Regularly persistent observers for bilinear system. Lecture Notes in Control and Information Sciences, Springer, 122, 130-140.

Calvet, J.-P., \& Arkun, Y. (1989). Stabilization of feedback linearized nonlinear processes under bounded perturbations. American Control Conference, Pittsburgh, PA, (pp. 747-752).

Castillo, F., Witrant, E., Prieur, C., \& Dugard, L. (2012). Dynamic boundary stabilization of linear and quasi-linear hyperbolic systems. IEEE Conference on Decision and Control CDC, Maui, HI, (pp. 29522957).

Cox, H.(1964). On the estimation of state variables and parameters for noisy dynamic systems.IEEE Transactions On Automatic Control, (9), 5-12.

Dufour, P., Flila, S., \& Hammouri, H. (2012). Observer design for MIMO non-uniformly observable systems. IEEE Transactions On Automatic Control, 57(2), 511-516.

Ellis, M., Durand, H., \& Christofides, P.D. (2014). A tutorial review of economic model predictive control methods. Journal of Process Control, 24 (8), 1156-1178. 
Flila, S., Dufour, P., \& Hammouri, H. (2008). Optimal input design for on-line identification: a coupled observer-MPC approach. IFAC World Congress, Seoul, South Korea, (pp. 11457-11462).

Forssell, U., \& Ljung, L. (1998). Identification of unstable systems using output error and box-jenkins model structures, in: Proceedings of the 29th IEEE CSS Chinese Control Conference, (pp. 3932-3937).

Franceschini, G., \& Macchietto, S., (2008). Optimal experiment design for linear systems with input-output constraints: State of the art. Chemical Engineering Science, 63, 4846-4872.

Gauthier, J.P., Hammouri, H., \& Othman, S. (1992). A simple observer for nonlinear systems applications to bioreactors. IEEE Transactions on Automatic Control, 37(6), 875-880.

Goodwin, G.C., \& Payne, R.L. (1977). Dynamic system identification: experiment design and data analysis. Mathematics in Science and Engineering, Vol. 136, Academic Press.

Hammouri, H. \& Morales, J.D.L (1990). Observer synthesis for state-affine systems. IEEE Conference on Decision and Control, Honolulu, HI, vol. 2, (pp. 784-785).

Huang, R., Harinath, E., \& Biegler, L.T., (2011). Lyapunov stability of economically oriented NMPC for cyclic processes. Journal of Process Control, 21 (4), 501-509.

Jain, H., Kaul, V., \& Ananthkrishnan, N. (2005). Parameter estimation of unstable, limit cycling systems using adaptive feedback linearization: example of delta wing roll dynamics. Journal of Sound and Vibration, 287, 939-960.

Jayasankar, B., Huang, B., \& Ben-Zv, A. (2010). Receding horizon experiment design with application in SOFC parameter estimation. Dynamics and Control of Process Systems, Leuven, Belgium, (pp. 527-532).

Keviczky, L. (1975). Design of experiments for the identification of linear dynamic systems. Technometrics, 17(3), 303-308.

Ljung, L. (1979). Asymptotic behavior of the extended kalman filter as a parameter estimator for linear systems, IEEE Transactions on Automatic Control, 24, 36-50.

Ljung, L. (1999). System identification: Theory for the user, (2nd ed.), Prentice Hall.

Nadri, M., Hammouri, H., \& Grajales, R. (2013). Observer design for uniformly observable systems with sampled measurements. IEEE Transactions on Automatic Control, 58(3), 757-762.

Nayfeh, A.H., Elzeba, J.M., \& Mook, D.T. (1989). Analytical study of the subsonic wing-rock phenomenon for slender delta wings. Journal of Aircraft, 26(9), 805-809.

Nelson, L., \& Stear, E. (1976). The simultaneous on-line estimation of parameters and states in linear systems, IEEE Transactions on Automatic Control, 21, 94-98.

Ng, T.S., Goodwin, G.C., \& Söderström, T. (1997). Optimal experiment design for linear systems with input-output constraints. Automatica, 13(6), 571-577.

Qian, J., Dufour, P., \& Nadri, M. (2013). Observer and model predictive control for on-line parameter identification in nonlinear systems. IFAC Dynamics and Control of Process Systems, Mumbai, India, (pp. 571-576).

Qian, J., Nadri, M., Moroşan, P.D.M., \& Dufour, P. (2014). Closed loop optimal experiment design for on-line parameter estimation. IFAC IEEE European Control Conference, Strasbourg, France, (pp. 1813-1818).

Rawlings, J.B., Angeli, D., \& Bates, C.N. (2012). Fundamentals of economic Model Predictive Control. IEEE Conference on Decision and Control, (pp. 3851-3861).

Walter, E., \& Pronzato, L. (1994). Identification of parametric models from experimental data, SpringerVerlag.

Willems, J.L. (1970). A general stability criterion for non-linear time-varying feedback systems. International Journal of Control, $11(4), 625-631$.

Zanon, M., Gros, S., \& Diehl, M. (2014). Indefinite linear MPC and approximated economic MPC for nonlinear systems. Journal of Process Control, 24(8), 1273-1281.

Zavala, V.M., \& Biegler, L.T., (2009). The advanced-step NMPC controller: Optimality, stability and robustness. Automatica, 45(1), 86-93.

Zhu, Y., \& Huang, B. (2011). Constrained receding-horizon experiment design and parameter estimation in the presence of poor initial conditions. AIChE Journal, 57(10), 2808-2820. 